\title{
Single Pulse Analysis of the Core-Dominated Pulsar $\mathrm{B} 0611+22$
}

\author{
Jeffrey S. Kern \\ Department of Physics, New Mexico Institute of Mining and \\ Technology, Socorro NM 87801 USA : jkern@nrao.edu
}

\begin{abstract}
Single pulse studies of pulsar radio emission provide a window into the time dependent behavior of the radio loud region. I have analyzed a series of precision polarimetric observations of pulsar B0611+22 to determine the geometry of the emission regio $n$. The observations are consistent with a central core emission region, and a periodically present conal component. This identification leads to the surprising result that all emission is from the leading half of the polar cap.
\end{abstract}

\section{Introduction}

Pulsars' radio emission region has long been associated with the polar cap region, however the details of the polar cap are not well understood. Single pulse studies provide a technique for investigating the time dependent geometry of the emission region. Single pulse analysis of the polarized emission from pulsar B0611+22 has allowed the development of a time dependent model for the radio emission region geometry. I find that the observations are consistent with a constant central region of core emiss ion and a periodically present conal component. Surprisingly this picture of the emission regions requires all of the emission to originate on the leading side of the polar cap.

\section{Analysis}

The data used in this project is carefully calibrated polarimetric data taken at Arecibo observatory. Using a $20 \mathrm{MHz}$ bandwidth and a time constant of $356-\mu \mathrm{s}$, the signal was sampled at $177-\mu$ s resolution. Because the signal to noise ratio was smal 1 , short ( $\sim 100$ period) averages were constructed. These short average profiles (SAPs) were then used for the rest of the analysis. The first order moment of the SAP total intensity distribution (FOM) was found to vary in a quasi-periodic way with $t$ ime. The characteristic frequency of this modulation is approximately 1200 pulses. Correlated with this modulation is a modulation of total intensity. Those SAPs with greater integrated total power tend to arrive earlier in phase, as measured by a FOM which is less than the mean.

Two long average profiles were created by averaging those SAPs which had either a significantly early or significantly late FOM. These profiles are represented by the error bars in figure 1. Gaussian fits were performed to quantify the differences betwe en the two profiles. A single Gaussian well describes the late 


\section{COMPONENT FITS TO SEPARATED PROFILES}

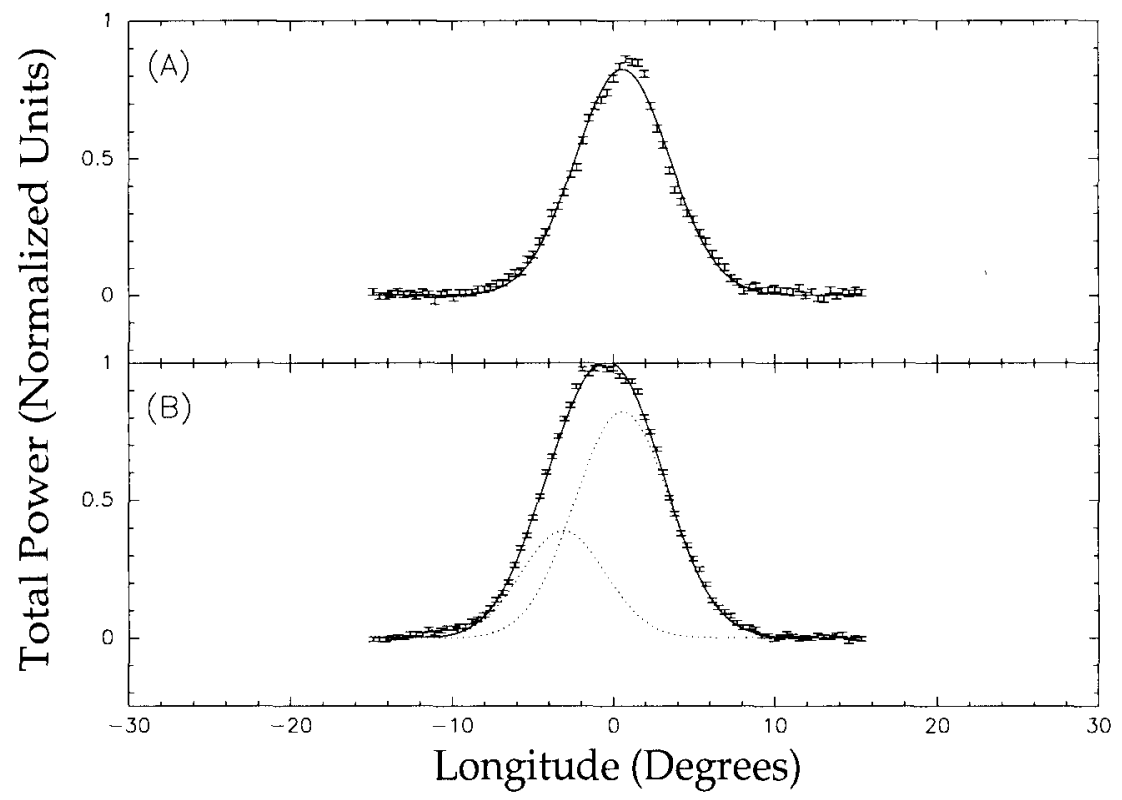

Figure 1. Component fits to the early and late profiles as described in the text.

profile (shown as the solid line in figure 1-A), while the early profile is well described by the addition of a second component on the leading side (the solid line in figure 1-B shows the to tal power while the dotted lines show the individual components).

Because of spectral considerations, and the polarization angle traverse I associate the constant later component with coral emission, and the periodically appearing leading component with a conal component. The quasi-periodic appearance of the conal comp onent can be understood as the Goldreich \& Julian (1969) $\mathbf{E} \times \mathbf{B}$ drift moving the emitting region in and out of our line of sight.

If the maximum rate of change in the PA traverse marks the center of the polar cap, then we are left with the remarkable result that all of the emission originates on the leading edge of the polar cap. This implies that the circulating conal emission reg ion must "turn off" on the trailing edge.

\section{References}

Goldreich, P. \& Julian, W. H. 1969, ApJ, 157, 869 